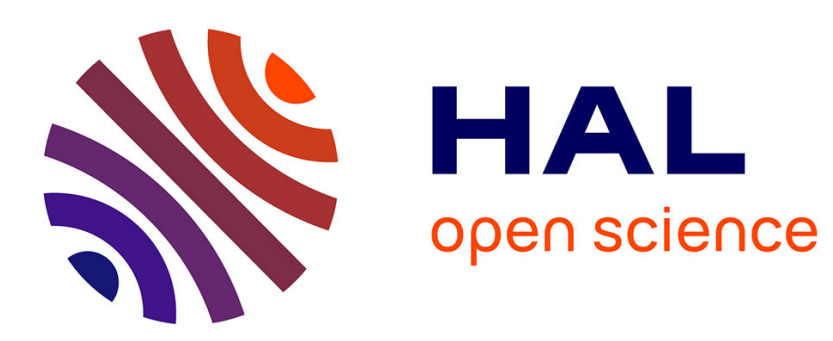

\title{
Stochastic binary problems with simple penalties for capacity constraints violations
}

\author{
Bernard Fortz, Martine Labbé, FranLouveaux, Michael Poss
}

\section{To cite this version:}

Bernard Fortz, Martine Labbé, FranLouveaux, Michael Poss. Stochastic binary problems with simple penalties for capacity constraints violations. Mathematical Programming, 2013, 138 (1-2), pp.199-221. hal-01255254

\section{HAL Id: hal-01255254 \\ https://hal.science/hal-01255254}

Submitted on 30 Mar 2021

HAL is a multi-disciplinary open access archive for the deposit and dissemination of scientific research documents, whether they are published or not. The documents may come from teaching and research institutions in France or abroad, or from public or private research centers.
L'archive ouverte pluridisciplinaire HAL, est destinée au dépôt et à la diffusion de documents scientifiques de niveau recherche, publiés ou non, émanant des établissements d'enseignement et de recherche français ou étrangers, des laboratoires publics ou privés. 


\title{
Stochastic binary problems with simple penalties for capacity constraints violations *
}

\author{
B. Fortz - M. Labbé - F. Louveaux - M. \\ Poss
}

Received: date / Accepted: date

\begin{abstract}
This paper studies stochastic programs with first-stage binary variables and capacity constraints, using simple penalties for capacities violations. In particular, we take a closer look at the knapsack problem with weights and capacity following independent random variables and prove that the problem is weakly $\mathcal{N} \mathcal{P}$-hard in general. We provide pseudo-polynomial algorithms for three special cases of the problem: constant weights and capacity uniformly distributed, subset sum with Gaussian weights and arbitrary random capacity, and subset sum with constant weights and arbitrary random capacity. We then turn to a branch-and-cut algorithm based on the outer approximation of the objective function. We provide computational results for the stochastic knapsack problem (i) with Gaussian weights and constant capacity and (ii) with constant weights and capacity uniformly distributed, on randomly generated instances inspired by computational results for the knapsack problem.
\end{abstract}

* This research is supported by an Actions de Recherche Concertées (ARC) project of the Communautée française de Belgique. Michael Poss is a research fellow of the Fonds pour la Formation à la Recherche dans l'Industrie et dans l'Agriculture (FRIA). Martine Labbé also acknowledges support from "Ministerio de Ciencia e Innovacion" of Spain through the research project MTM2009-14039-C06.

B. Fortz

Department of Computer Science, Faculté des Sciences, Université Libre de Bruxelles, Brussels, Belgium

E-mail: bfortz@ulb.ac.be

M. Labbé

Department of Computer Science, Faculté des Sciences, Université Libre de Bruxelles, Brussels, Belgium.

E-mail: mlabbe@ulb.ac.be

F. Louveaux

Department of Business Administration, University of Namur (FUNDP), Namur, Belgium.

E-mail: flouveau@fundp.ac.be

M. Poss

Department of Computer Science, Faculté des Sciences, Université Libre de Bruxelles, Brussels, Belgium.

E-mail: mposs@ulb.ac.be 
Keywords Stochastic programming · Knapsack problem · Pseudo-polynomial algorithm · Mixed-integer non-linear programming · Branch-and-cut algorithm.

\section{Introduction}

An important class of optimization problems with binary variables involves capacity constraints. In some of these problems, we must transport a set of goods from suppliers to customers without exceeding the capacity of transporting units. In others, we must prescribe a production plan that respects the capacity of producing units. An important subproblem that arises in both examples is how to fill the capacity of each transporting (or producing) unit in the best way, leading to the knapsack problem. Many efficient solution methods for capacitated problems benefit from fast algorithms for the knapsack problem, for instance, by generating cover cuts or by providing strong bounds within decomposition algorithms, see $[3,25]$, among others. In what follows, we work more specifically with uncertain parameters.

Different frameworks are able to handle capacity constraints with parameters following random variables. Probabilistic constraints are convenient to use because they do not interfere with the structure of the original model. However, they yield very difficult optimization problems, although recent works have proposed solution methods for integer programs with probabilistic constraints $[4,16]$. Herein we study a different framework, where additional capacity can be added at a given unitary cost, yielding a stochastic program with linear simple-recourse. This leads to tractable reformulations, as long as we can handle the multi-variate integral involved in the recourse function. Efficient solution methods for stochastic linear programs with simple-recourse have been developed in the past, see for instance [30]. The case of integer recourse has also been studied extensively $[11,21]$.

In this paper, we apply simple penalty recourse to the difficult class of binary problems with capacity constraints. We look more specifically at the stochastic knapsack problem and derive two types of results. First, we prove that three special cases of the stochastic knapsack problem are weakly $\mathcal{N} \mathcal{P}$-hard:

- Stochastic knapsack with fixed weights and uniformly distributed capacity.

- Stochastic subset sum with Gaussian weights and arbitrary random capacity.

- Stochastic subset sum with fixed weights and arbitrary random capacity.

Then, we show that the LP/NLP algorithm of Quesada and Grossman [28] enables us to solve efficiently the stochastic knapsack problem with Gaussian random variables. Our computational results show that this algorithm is able to solve in less than a minute problems involving up to a few thousands of variables, outperforming previous works on the problem $[10,19]$. Finally, we compare the pseudo-polynomial algorithms and the LP/NLP algorithm on the special cases. It turns out that some of the stochastic subset sum problems can be solved extremely fast by using the specialized algorithm from Pisinger [26].

The paper is structure as follows. Section 2 introduces the general models studied herein and provides some examples, including the stochastic knapsack problem. Section 3 studies the complexity of the special cases of the stochastic knapsack problem, providing pseudo-polynomial algorithms. Section 4 presents an algorithm for the general models and provide extensive computational experiments. A comparison of the general algorithm and the pseudo-polynomial approaches is also presented for the special cases. The paper concludes with Section 5 . 


\section{The models}

\subsection{Capacity constraints}

In this paper, we study binary problems featuring capacity constraints, that is

$$
\sum_{i=1}^{n} a_{i} x_{i} \leq a_{0}
$$

where $a_{i}$ and $a_{0}$ are positive numbers. We denote such "capacitated problems" by (CAP) in what follows. When coefficients $a_{i}$ and $a_{0}$ are random variables, (1) yields one equation for each scenario in a set of scenarios $\Omega$, which is not convenient when $\Omega$ contains a large (sometimes infinite) number of scenarios or when some of the probability weights are very small. Replacing (1) by the probabilistic constraint

$$
P\left(\sum_{i=1}^{n} a_{i} x_{i} \leq a_{0}\right) \geq 1-\epsilon
$$

for $\epsilon \in(0,1)$ is thus a natural choice in the stochastic context. Although easy to incorporate into models, probabilistic constraints yield, in general, very difficult optimization problems. Moreover, they neglect the amount by which the constraint is violated while this can be crucial in some applications. For instance, electrical lines are built to resist against higher power flow than expected for a reasonable period of time, while an extremely high power flow even for a short period of time is likely to burn some of the lines. These two problems can be handled by the introduction of a penalty $y$ for capacity violation. Then, (1) becomes

$$
\sum_{i=1}^{n} a_{i}(\omega) x_{i} \leq a_{0}(\omega)+y(\omega) \quad \omega \in \Omega,
$$

and the weighted expected violation of the capacity $K \mathbb{E}_{a}[y(\omega)]$ is subtracted from the objective function (for a maximization problem), where $\mathbb{E}_{a}[y(\omega)]=\int_{A} y(a) d F_{a}(a)$ and $F_{a}: \mathbb{R}^{n+1} \rightarrow \mathbb{R}_{+}$is the distribution function of the random vector $a=\left(a_{0}, a_{1}, \ldots, a_{n}\right)$ : $\Omega \rightarrow A \subset \mathbb{R}^{n+1}$. This simple-recourse formulation weights capacity violation through parameter $K$ and yields tractable optimization problems, thus addressing the two drawbacks of probabilistic constraints mentioned above. In what follows, we denote by (SCAP) problems featuring penalized capacity constraints (3).

Consider that the original objective function is $\sum_{i=1}^{n} c_{i} x_{i}$. Because constraints (3) are satisfied at equality in any optimal solution with $y(\omega)>0$, variable $y(\omega)$ can be replaced with $\max \left(0, \sum_{i=1}^{n} a_{i}(\omega) x_{i}-a_{0}(\omega)\right)$, resulting in the non-linear objective function:

$$
\sum_{i=1}^{n} c_{i} x_{i}-K \mathbb{E}_{a}\left[\max \left(0, \sum_{i=1}^{n} a_{i}(\omega) x_{i}-a_{0}(\omega)\right)\right]
$$

We review next two examples of (SCAP) that have been previously studied in the literature. 


\subsubsection{Traveling salesman}

Given a complete directed graph with node set $N$, and travel $\operatorname{costs} c_{i j}, i, j \in N$, the traveling salesman problem (TSP) looks for a cycle of minimum cost passing exactly once through each node. A generalization of the problem considers that a time limit $T$ is given and that each edge has a travel time. The obtained tour can not exceed the time limit. The stochastic extension written below describes each travel time by a random variable $a_{i j}$. Whenever the total travel time of a tour exceeds $T$, a penalty must be paid at the cost of $K$ per unit of delay. This results in the subsequent problem, which has been extended to a three-index model by Laporte et al. [20]:

$$
\begin{array}{llc}
\min & \sum_{i, j \in N} c_{i j} x_{i j}+K \mathbb{E}_{a}\left[\max \left(0, \sum_{i, j \in N} a_{i j}(\omega) x_{i j}-T\right)\right] \\
\text { s.t. } & \sum_{j \in N} x_{i j}=1 & i \in N \\
& \sum_{i \in N} x_{i j}=1 & j \in N \\
& \sum_{i, j \in S} x_{i j} \leq|S|-1 & \\
& x_{i j} \in\{0,1\} . &
\end{array}
$$

The only difference between the problem above and the deterministic (TSP) is the presence of a non linear term in the objective.

\subsubsection{Elastic generalized assignment}

The generalized assignment problem looks for optimal assignment of $n$ jobs to $m$ machines that have a limited capacity. The problem has numerous applications, including fixed-charge plant location models and scheduling of tasks in servers, among others. Being $\mathcal{N} \mathcal{P}$-hard, the problem has attracted a considerable amount of attention in the past, leading to efficient solution methods, see [9] and [25], among others. Brown and Graves [8] introduce penalties for unsatisfied capacity constraints, yielding the elastic generalized assignment problem. Spoerl and Wood extended the latter to stochastic settings in [29], yielding

$$
\begin{aligned}
& \min \sum_{i=1}^{n} \sum_{j=1}^{m} c_{i j} x_{i j}+\sum_{j=1}^{m} K_{j} \mathbb{E}_{a}\left[\max \left(0, \sum_{i=1}^{n} a_{i} x_{i j}-a_{0 j}\right)\right] \\
& \text { (SEGAP) } \quad \sum_{j=1}^{m} x_{i j}=1 \quad i=1, \ldots, n \\
& x_{i j} \in\{0,1\} \text {. }
\end{aligned}
$$

The resulting (SEGAP) presents the structure of the assignment problem with a nonlinear cost function. 
2.2 The knapsack problem

Given a set of items N, the deterministic knapsack problem looks for a subset of N not exceeding the capacity $a_{0}$ of the knapsack and maximizing the total profit. Each item $i \in \mathrm{N}$ has a profit $p_{i}$ and a weight $a_{i}$. Our two-stage stochastic version of the problem considers that weights $a_{i}(\omega)$ and capacity $a_{0}(\omega)$ are independent random variables. The subset of items is chosen first, then any real amount of additional capacity can be bought at the unitary price of $K$, depending on the scenario $\omega$. The objective function maximizes the profit of the chosen subset minus the expected cost of the additional capacity:

$$
\begin{array}{ll}
\max & \sum_{i \in \mathrm{N}} p_{i} x_{i}-K \mathbb{E}_{a}\left[\max \left(0, \sum_{i \in \mathrm{N}} a_{i}(\omega) x_{i}-a_{0}(\omega)\right)\right] \\
\text { s.t. } & x_{i} \in\{0,1\} .
\end{array}
$$

This problem can be thought of as the following resource allocation problem [15]. A decision maker has to choose a subset of $n$ known alternative projects to take on. For this purpose, a known quantity $a_{0}$ of relatively low-cost resource is available to be allocated. Any additional amount of resource required can be obtained at a known incremental cost of $K$ per unit of resource. The amount $a_{i}$ of resource required by each project is not known at the time the decision has to be made, but we assume that the decision maker has an estimate of the probability distribution of those $a_{i}$. Finally, each project $i$ has an expected reward of $p_{i}$.

(SKP) has been first formulated by Cohn and Barnhart [10] who consider that $a_{0}$ is given and that $a_{i}$ follow Gaussian variables. They derive basic properties and propose a simple branch-and-bound algorithm that they test on an example with 15 variables. Recently, Kosuch and Lisser [19] use a stochastic gradient method to solve (SKP) with Gaussian variables. They solve the problem up to 150 variables in at most two hours. Kleywegt et al. [15] work on a similar model with discrete random variables to test their sample average approximation method. Other ways of considering uncertainties in the parameters of the knapsack problem include knapsack with a probabilistic constraint $[18,23]$, robust knapsack [17] and dynamic knapsack [14], among others.

\section{Pseudo-polynomial cases}

In this section we show that Problem (SKP) is in general weakly $\mathcal{N} \mathcal{P}$-hard. We then turn to special cases of (SKP) that can be solved in pseudo-polynomial time. In the remainder of this section, we use the notations $B$ and $\mathcal{B}$ to denote $\{0,1\}^{n}$ and $[0,1]^{n}$, respectively; the summation $\sum$ refers to the sum over $N$ unless stated otherwise.

Proposition 1 Problem (SKP) is $\mathcal{N} \mathcal{P}$-hard.

Proof We present a polynomial reduction from the problem of solving the deterministic knapsack to optimality to the problem of solving (SKP) to optimality. Given an instance of the deterministic knapsack problem described by the positive integer parameters $a_{0}$ and $p_{i}, a_{i}$ for each $i \in \mathrm{N}$, we build an instance of (SKP) with a unique scenario $\Omega=\{\omega\}$, 
$a_{0}(\omega)=a_{0}, p_{i}(\omega)=p_{i}$ and $a_{i}(\omega)=a_{i}$ for each $i \in \mathrm{N}$, and $K=\max _{i \in \mathrm{N}} p_{i}+1$. Writing the capacity constraint of (SKP) explicitly, the problem reads:

$$
\max _{x \in \mathrm{B}, y \geq 0}\left\{\sum_{i \in \mathrm{N}} p_{i}(\omega) x_{i}-K y(\omega) \text { s.t. } \sum_{i \in \mathrm{N}} a_{i}(\omega) x_{i} \leq a_{0}+y(\omega)\right\} .
$$

We show next that $y(\omega)$ is equal to 0 in any optimal solution, so that (6) is equivalent to the instance of the deterministic knapsack problem. To see this is true, consider a feasible solution for (6) where $y(\omega)>0$. The integrality requirements on $a(\omega)$ imply that $y(\omega) \geq 1$. Hence, withdrawing any item from the knapsack improves the objective function, because it decreases the penalty by at least $\max _{i \in \mathrm{N}} p_{i}+1$ while decreasing the profit by at most $\max _{i \in \mathrm{N}} p_{i}$.

3.1 Fixed weights and uniformly distributed capacity

In this section, we consider that weights $a_{i}$ are fixed so that (SKP) becomes:

$$
\max _{x \in \mathrm{B}} \sum p_{i} x_{i}-K \int_{0}^{\sum a_{i} x_{i}}\left(\sum a_{i} x_{i}-a_{0}\right) d F\left(a_{0}\right) .
$$

Assuming that $a_{0}$ is uniformly distributed between positive integers $a_{0}$ and $\overline{a_{0}}$ and that all parameters are integers we show next that the optimization problem can be solved by a pseudo-polynomial algorithm under a mild assumption on its parameters. Note that this problem can also be seen as a robust knapsack problem with linear penalty [24]. With $a_{0}$ uniformly distributed, (7) becomes:

$$
\max _{x \in \mathrm{B}} \sum p_{i} x_{i}-\frac{K}{\overline{a_{0}}-\underline{a_{0}}} \int_{\min \left(\sum a_{i} x_{i}, \underline{a_{0}}\right)}^{\min \left(\sum a_{i} x_{i}, \overline{a_{0}}\right)}\left(\sum a_{i} x_{i}-a_{0}\right) d a_{0},
$$

where we assume $\sum a_{i}>\underline{a_{0}}$ to avoid the trivial solution $x=(1, \ldots, 1)$.

Theorem 1 Problem (8) is $\mathcal{N} \mathcal{P}$-hard. Moreover, if $\left(p_{i}+\frac{K a_{i}}{2\left(\overline{a_{0}}-\underline{a_{0}}\right)}\left(2 \underline{a_{0}}-a_{i}\right)\right) \geq 0$ for each $i \in \mathrm{N}$, the problem can be solved in $O\left(n K \sum a_{i}\right)$.

For a large number of items with individual volumes small enough, this condition is likely to be satisfied.

Lemma 1 Problem (8) is $\mathcal{N} \mathcal{P}$-hard.

Proof Consider an instance of the deterministic knapsack problem with positive integer $a_{0}, a_{i}$ and $p_{i}$ for each $i \in \mathrm{N}$. We show that the optimal solutions of this problem are the optimal solutions of an instance of problem (8) with $\overline{a_{0}}=a_{0}+1=a_{0}$ and $K=2 \max _{i \in \mathrm{N}} p_{i}+1$. Let $x^{*} \in \mathrm{B}$ and suppose that $\sum a_{i} x_{i}^{*}>a_{0}$. Then, because $a$ is integer it holds that $\sum a_{i} x_{i}^{*} \geq a_{0}+1=\overline{a_{0}}$. Withdrawing any object $j \in \mathrm{N}$ from the knapsack decreases the penalty by

$$
K \int_{\underline{a_{0}}}^{\overline{a_{0}}}\left(\sum_{i \in \mathrm{N}} a_{i} x_{i}^{*}-\sum_{i \in \mathrm{N} \backslash\{j\}} a_{i} x_{i}^{*}\right) d a_{0} \geq K \int_{\underline{a_{0}}}^{\overline{a_{0}}} d a_{0}=K / 2=\max _{i \in \mathrm{N}} p_{i}+1 / 2,
$$

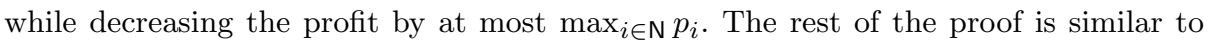
the proof of Proposition 1. 
The remainder of the section shows how (8) can be solved in pseudo-polynomial time whenever $\left(p_{i}+\frac{K a_{i}}{2\left(\overline{a_{0}}-\underline{a_{0}}\right)}\left(2 \underline{a_{0}}-a_{i}\right)\right) \geq 0$ for each $i \in \mathrm{N}$. Let $Z$ be the the function to be maximized in (8). $\overline{\mathrm{We}}$ rewrite $Z$ as follows:

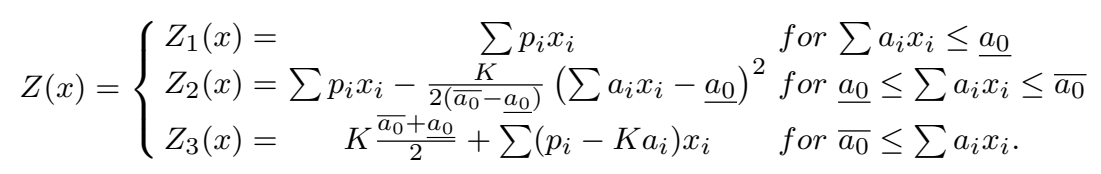

Lemma 2 Let $x^{*}, x_{1}^{*}, x_{2}^{*}$ and $x_{3}^{*}$ be optimal solutions of $\max _{\mathrm{B}} Z(x)$, $\max _{\mathrm{B}}\left\{Z_{1}(x)\right.$ s.t. $\left.\sum a_{i} x_{i} \leq \underline{a_{0}}\right\}, \max _{\mathrm{B}} Z_{2}(x)$ and $\max _{\mathrm{B}}\left\{Z_{3}(x)\right.$ s.t. $\left.\sum a_{i} x_{i} \geq \overline{a_{0}}\right\}$, respectively. Then, $Z\left(x^{*}\right)=\max \left(Z_{1}\left(x_{1}^{*}\right), Z_{2}\left(x_{2}^{*}\right), Z_{3}\left(x_{3}^{*}\right)\right)$.

Proof We can relax the domain restriction $\underline{a_{0}} \leq \sum a_{i} x_{i} \leq \overline{a_{0}}$ for $Z_{2}$ because $Z_{1}(x) \geq$ $Z_{2}(x)$ and $Z_{3}(x) \geq Z_{2}(x)$ for any $x \in \mathcal{B}$.

The following three lemmas show that each of the problems from Lemma 2 can be solved in pseudo-polynomial time.

Lemma 3 Maximizing $Z_{1}(x)$, for $x$ binary and $\sum a_{i} x_{i} \leq \underline{a_{0}}$, can be done in $O\left(n \sum a_{i}\right)$.

Proof This is a knapsack problem, which can be optimized in $O\left(n \underline{a_{0}}\right)$ and thus in $O\left(n \sum a_{i}\right)$ because $\underline{a_{0}}<\sum a_{i}$.

Lemma 4 Maximizing $Z_{3}(x)$, for $x$ binary and $\sum a_{i} x_{i} \geq \overline{a_{0}}$, can be done in $O\left(n \sum a_{i}\right)$.

Proof In the following, we assume that $\sum a_{i}>\overline{a_{0}}$, otherwise $\sum a_{i} x_{i}$ is always smaller than $\overline{a_{0}}$ so that the problem does not have a solution. We show that the problem is a knapsack problem.

1. Define $\mathrm{M}=\left\{i \in \mathrm{N} \mid p_{i}-K a_{i}<0\right\}$ and $\tilde{a_{0}}=\sum a_{i}-\overline{a_{0}}$; let $x^{*}$ be the solution to the following knapsack problem $\left(x_{i}^{*}=0\right.$ for $\left.i \in \mathrm{N} / \mathrm{M}\right)$

$$
\max _{\mathrm{B}}\left(\sum_{i \in \mathrm{M}}\left(K a_{i}-p_{i}\right) x_{i} \quad \text { s.t } \quad \sum_{i \in \mathrm{M}} a_{i} x_{i} \leq \tilde{a_{0}}\right) .
$$

2. An optimal solution to $\max _{\mathrm{B}}\left\{Z_{3}(x)\right.$ s.t. $\left.\sum a_{i} x_{i} \geq \overline{a_{0}}\right\}$ is given by $x_{i}=1-x_{i}^{*}$, for each $i \in \mathrm{N}$.

Then, because $\tilde{a_{0}} \leq \sum a_{i},(10)$ can be solved in $O\left(n \sum a_{i}\right)$.

Lemma 5 If $\left(p_{i}+\frac{K a_{i}}{2\left(\overline{a_{0}}-\underline{a_{0}}\right)}\left(2 \underline{a_{0}}-a_{i}\right)\right) \geq 0$ for each $i \in \mathrm{N}$, maximizing $Z_{2}(x)$, for $x$ binary, can be done in $O\left(\overline{n K} \sum a_{i}\right)$.

Proof Expanding the square in $Z_{2}$, and using the identity $x_{i}^{2}=x_{i}$ because $x \in \mathrm{B}$, we obtain:

$$
-\frac{K{\underline{a_{0}}}^{2}}{2\left(\overline{a_{0}}-\underline{a_{0}}\right)}+\max _{x \in \mathrm{B}}\left(\sum_{i \in \mathrm{N}}\left(p_{i}+\frac{K a_{i}}{2\left(\overline{a_{0}}-\underline{a_{0}}\right)}\left(2 \underline{a_{0}}-a_{i}\right)\right) x_{i}-\frac{K}{\overline{a_{0}}-\underline{a_{0}}} \sum_{\substack{i, j \in \mathrm{N} \\ i \neq j}} a_{i} a_{j} x_{i} x_{j}\right) .
$$


Assuming that $\left(p_{i}+\frac{K a_{i}}{2\left(\overline{a_{0}}-\underline{a_{0}}\right)}\left(2 \underline{a_{0}}-a_{i}\right)\right) \geq 0$ for each $i \in \mathrm{N}$, linear coefficients of (11) are all positive. We need them to be integer as well to apply the tools from optimization of pseudo boolean functions.

Multiplying all terms by $4 K\left(\overline{a_{0}}-\underline{a_{0}}\right),(11)$ becomes a particular case of halfproducts [2]

$$
f=\sum_{i \in \mathrm{N}} c_{i} x_{i}-\sum_{\substack{i, j \in \mathrm{N} \\ i \neq j}} a_{i} b_{j} x_{i} x_{j},
$$

where $c \mapsto 4 K\left(\overline{a_{0}}-\underline{a_{0}}\right)\left(p+\frac{K w}{2\left(\overline{a_{0}}-\underline{a_{0}}\right)}\left(2 \underline{a_{0}}-a_{i}\right)\right)$ and $a=b \mapsto 2 K w$. Badics and Boros [2] provide a dynamic programming algorithm for general half-products with positive coefficients. Its running time is $O(n A)$, where $A=2 K \sum a_{i}$.

Besides this dynamic programming approach, new versions of optimization softwares, including CPLEX 11, can manage maximization of integer problems with a concave and quadratic objective function. Nevertheless, we show in Section 4.2 that the LP/NLP algorithm described in the Section 4.1.1 solves (11) much faster than CPLEX 11 and the algorithm from Badics and Boros do.

\subsection{Subset sum}

A well known particular case of the deterministic knapsack problem is the subset sum problem which assumes that weight $a_{i}$ is equal to profit $p_{i}$ for each item $i \in \mathrm{N}$. Even though weakly $\mathcal{N} \mathcal{P}$-hard to solve, adapted algorithms can have a much better behavior for this problem than for the general knapsack.

To the best of our knowledge, no stochastic version of the subset sum has been addressed in the literature so far. To define the stochastic subset sum, we replace the deterministic constraint $a=p$ by:

$-\mathbb{E}_{a}[a]=p$,

$-\operatorname{Var}[a]=\lambda p$ for some $\lambda \geq 0$,

where $\mathbb{E}_{a}[v]=\left(\mathbb{E}_{a}\left[v_{1}\right], \ldots, \mathbb{E}_{a}\left[v_{n}\right]\right)$ and $\operatorname{Var}[v]=\left(\operatorname{Var}\left[v_{1}\right], \ldots, \operatorname{Var}\left[v_{n}\right]\right)$ for any random vector $v$. The constraint $\mathbb{E}_{a}[a]=p$ is the direct extension of $a=p$. Then, to enforce the link between $p$ and $a$ we also impose $\operatorname{Var}[a]=\lambda p$. Note that the case $\lambda=0$ results in a deterministic knapsack, where additional capacity can be purchased at the incremental cost of $K$ per unit, see problem (6).

The relation $\operatorname{Var}[a]=\lambda \mathbb{E}_{a}[a]$ is not invariant under the change of units of measurement. Therefore, for a given problem instance, the units used in the parameters definition should be chosen carefully when constructing the mathematical model. In particular, whenever $|\mathrm{N}|=1$ the units can always be scaled so that the relation is satisfied. This linear relation has been used previously for (SEGAP) through the Proportional Mean-Variance Model [29].

\subsubsection{Gaussian weights}

In this section, we assume that weights $a_{i}, i \in \mathrm{N}$, are independent Gaussian variables with parameters $\mu_{i}$ and $\sigma_{i}^{2}=\lambda \mu_{i}, i \in \mathrm{N}$, for some $\lambda \geq 0$, and that capacity $a_{0}$ is a strictly positive random variable whose smallest value is denoted by $\underline{a_{0}}>0$. 
This is motivated by the following summation property: if $a_{1}, \ldots, a_{n}$ are independent Gaussians of mean $\mu_{i}$ and variance $\sigma_{i}^{2}$, and $x_{i}$ are real numbers, then $Y:=\sum x_{i} a_{i} \sim$ $\mathcal{N}\left(\mu(x), \sigma^{2}(x)\right)$, with $\mu(x)=\sum x_{i} \mu_{i}$ and $\sigma^{2}(x)=\sum x_{i}^{2} \sigma_{i}^{2}$. Moreover, Gaussians are often used to represent the error made on estimations of parameters for many physical and economical problems.

Theorem 2 Consider the problem

$$
\max _{x \in \mathrm{B}} \sum \mu_{i} x_{i}-K \mathbb{E}_{a}\left[\max \left(0, \sum a_{i}(\omega) x_{i}-a_{0}(\omega)\right)\right]
$$

where $a_{i} \sim \mathcal{N}\left(\mu_{i}, \lambda \mu_{i}\right), \mu$ is an integer vector and $a_{0}$ is a strictly positive random variable. Problem (12) is weakly $\mathcal{N} \mathcal{P}$-hard and can be solved by a pseudo-polynomial algorithm in $O\left(n \sum \mu_{i}\right)$.

The fact that (12) is $\mathcal{N} \mathcal{P}$-hard easily follows from an argument similar to the one used in Proposition 1, taking $\lambda=0, K=\max _{i \in \mathrm{N}} \mu_{i}+1$, and $a_{0}>0$ as a constant random variable.

The rest of the section shows how to construct a pseudo-polynomial algorithm for (12). Let $a(x)=\sum a_{i} x_{i} \sim \mathcal{N}\left(\mu(x), \sigma^{2}(x)\right)$, so that the usual recourse function reads

$$
\mathcal{Q}(x)=-K \mathbb{E}_{a}\left[\max \left(0, a(x ; \omega)-a_{0}(\omega)\right)\right]
$$

and consider the auxiliary function

$$
\mathcal{R}(x)=-K \mathbb{E}_{a}\left[\max \left(0, \hat{a}(x ; \omega)-a_{0}(\omega)\right)\right],
$$

where $\hat{a}(x) \sim \mathcal{N}\left(\mu(x), \hat{\sigma}^{2}(x)\right)$, with $\mu(x)=\sum \mu_{i} x_{i}$ as before and $\hat{\sigma}^{2}(x)=\sum \sigma_{i}^{2} x_{i}$. Note that for each $i \in \mathrm{N}, x_{i}^{2}=x_{i}$ when $x \in \mathrm{B}$ so that $\mathcal{Q}(x)=\mathcal{R}(x)$ when $x \in \mathrm{B}$.

We define then $Z_{\mathcal{Q}}(x)=\sum \mu_{i} x_{i}+\mathcal{Q}(x)$ and $Z_{\mathcal{R}}(x)=\sum \mu_{i} x_{i}+\mathcal{R}(x)$, so that functions $Z_{\mathcal{Q}}(x)$ and $Z_{\mathcal{R}}(x)$ coincide on $\mathrm{B}$, and $\max _{\mathrm{B}} Z_{\mathcal{Q}}(x)=\max _{\mathrm{B}} Z_{\mathcal{R}}(x)$. In what follows, we focus on the maximization of $Z_{\mathcal{R}}$. This is motivated by the following property:

Lemma 6 If $\sigma_{i}^{2}=\lambda \mu_{i}$ for each $1 \leq i \leq n$ and some $\lambda \geq 0$, then there exists a function $\hat{Z}:\left[0, \sum \mu_{i}\right] \rightarrow \mathbb{R}$ such that for all $x \in \mathcal{B}, Z_{\mathcal{R}}(x)=\hat{Z}(z)$ with $z=\sum \mu_{i} x_{i}$.

Proof By definition of $z, Z_{\mathcal{R}}$ can be rewritten as $z+\mathcal{R}(x)$. Then, we see that $\mu(x)=$ $\sum \mu_{i} x_{i}=z$ and $\hat{\sigma}(x)=\sqrt{\sum \sigma_{i}^{2} x_{i}}=\sqrt{\lambda \sum \mu_{i} x_{i}}=\sqrt{\lambda z}$ proving the result.

Note that $\mathcal{R}(x)=\mathcal{Q}(x)$ only when $x \in \mathrm{B}$; when $x \in \mathcal{B} / \mathrm{B}$ these functions may be different. In particular, neither $Z_{\mathcal{R}}$ nor $\hat{Z}$ inherit from the concavity of recourse functions [5]. Nevertheless, we prove analytically that $\hat{Z}$ is concave, assuming that $\lambda^{2} / 4 a_{0} \leq 1$, where $\underline{a_{0}}$ is the smallest value taken by $a_{0}$. This assumption is not restrictive because given an instance of the problem where $\alpha:=\lambda^{2} / 4 a_{0}>1$, one can always scale the data so that the assumption is satisfied. Namely, suppose that the current unit choice for the data $a$ yields a ratio $\alpha>1$. Then, expressing the data in a new unit so that $a^{\prime}=a / \alpha$, we see that the ratio $\lambda^{2} / 4 \underline{a_{0}}$ is equal to one.

Lemma 7 If $\lambda^{2} / 4 a_{0} \leq 1$, the function $\hat{Z}$ is concave on its domain. 
Proof Let $F_{0}$ be the distribution function of $a_{0}$. If $\lambda=0, \hat{Z}$ is defined by

$$
z-K \int_{\underline{a_{0}}}^{\infty}\left(z-a_{0}\right) d F_{0}\left(a_{0}\right)
$$

and its second derivative is null. Hence, we suppose that $\lambda>0$ in the following and we let $f$ be the density function of $\mathcal{N}(0,1) . \hat{Z}$ is defined by the following expression

$$
\hat{Z}(z)=z-K \int_{\underline{a_{0}}}^{\infty}\left\{\frac{1}{\lambda z} \int_{0}^{\infty} f\left(\frac{a-z+a_{0}}{\lambda z}\right) d a\right\} d F_{0}\left(a_{0}\right) .
$$

Following [10], among others, the inner integral can be simplified, yielding:

$$
\hat{Z}(z)=z-K \int_{\underline{a_{0}}}^{\infty}\left\{\lambda z f\left(\frac{a_{0}-z}{\lambda z}\right)+\left(z-a_{0}\right) G\left(\frac{a_{0}-z}{\lambda z}\right)\right\} d F_{0}\left(a_{0}\right),
$$

where $G=1-\Phi$ and $\Phi$ is the distribution function of $\mathcal{N}(0,1)$. Computing the second derivative of (14) for any $z>0$, we obtain:

$$
\hat{Z}^{\prime \prime}(z)=-\frac{K}{8 \lambda \sqrt{\pi}} \int_{\underline{a_{0}}}^{\infty}\left\{\left(\lambda^{-2}\left(z+a_{0}\right)^{2}-z\right) \frac{e^{-\frac{\left(z-a_{0}\right)^{2}}{2 \lambda^{2} z}}}{\sqrt{z}^{5}}\right\} d F_{0}\left(a_{0}\right) .
$$

Because $F_{0}$ can be arbitrarily chosen, (15) is non-positive if and only if its integrand is non-negative for all $a_{0} \geq a_{0}>0$. Since the second factor of the integrand is always positive, we must ensure that

$$
\lambda^{-2}\left(z+a_{0}\right)^{2}-z=z^{2} \lambda^{-2}+z\left(2 a_{0} \lambda^{-2}-1\right)+a_{0}^{2} \lambda^{-2}
$$

is non-negative for all $a_{0} \geq \underline{a_{0}}>0$. This is the case whenever $\lambda^{2} / 4 a_{0} \leq 1$. Hence, since (16) is an increasing function of $a_{0}$, we see that $\hat{Z}^{\prime \prime}(z)$ is non-positive whenever $\lambda^{2} / 4 a_{0} \leq 1$.

In the following we consider that the units have been scaled by factor $\alpha=\lambda^{2} / 4 \underline{a_{0}}$. We rename the vector of means as $\nu$ so that $\nu=\mu / \alpha$ where $\mu$ is integer.

Thanks to Lemma 7 , the set of the maximizers of $\hat{Z}$ is connected. Suppose that we can compute a maximizer $z^{*}$ of $\hat{Z}$ over $\mathbb{R}^{+}$, which may be greater than $\sum \nu_{i}$. The concavity of $\hat{Z}$ implies that

$$
z_{1} \leq z_{2} \leq z^{*} \Rightarrow \hat{Z}\left(z_{1}\right) \leq \hat{Z}\left(z_{2}\right) \quad \text { and } \quad z^{*} \leq z_{2} \leq z_{1} \Rightarrow \hat{Z}\left(z_{1}\right) \leq \hat{Z}\left(z_{2}\right),
$$

for any $z_{1}, z_{2} \in\left[1, \sum \nu_{i}\right]$. Recalling that $\hat{Z}\left(\sum \nu_{i} x_{i}\right)=Z_{\mathcal{R}}(x)$, we can write similar inequalities for $Z_{\mathcal{R}}$ :

$$
\begin{aligned}
\sum \nu_{i} x_{1 i} \leq \sum \nu_{i} x_{2 i} \leq z^{*} \Rightarrow Z_{\mathcal{R}}\left(x_{1}\right) & \leq Z_{\mathcal{R}}\left(x_{2}\right) \\
\text { and } \quad z^{*} \leq \sum \nu_{i} x_{2 i} \leq \sum \nu_{i} x_{1 i} \Rightarrow Z_{\mathcal{R}}\left(x_{1}\right) & \leq Z_{\mathcal{R}}\left(x_{2}\right),
\end{aligned}
$$

for any fractional vectors $x_{1}, x_{2} \in \mathcal{B}$.

Hence, the closer $\sum \nu_{i} x_{i}$ is to $z^{*}$, the higher is $Z_{\mathcal{R}}(x)$. Then, two situations can happen. If $\sum \nu_{i} \leq z^{*}$, the closest $z=\sum \nu_{i} x_{i}, x \in \mathrm{B}$, to $z^{*}$ is given by $x_{i}^{*}=1$ for each $i \in \mathrm{N}$. This $x^{*}$ is the solution to (12). If $\sum \nu_{i}>z^{*}$, we must look for $x_{1}^{*}$ and $x_{2}^{*}$ in $\mathrm{B}$ that minimize the distances between $\sum \nu_{i} x_{j i}^{*}$ and $z^{*}$, where $\sum \nu_{i} x_{1 i}^{*} \leq z^{*}$ and 
$\sum \nu_{i} x_{2 i}^{*} \geq z^{*}$. In fact, because $\nu=\mu / \alpha$ and $\mu$ is integer, it is sufficient to compute $\left\lceil\alpha z^{*}\right\rceil$ and $\left\lfloor\alpha z^{*}\right\rfloor$, and the vectors $x_{1}^{*}$ and $x_{2}^{*}$ are optimal solutions of the following two subset sum problems:

$$
\begin{array}{ll}
\max & \sum_{i \in \mathrm{N}} \mu_{i} x_{i} \\
\text { s.t. } & \sum \mu_{i} x_{i} \leq\left\lfloor\alpha z^{*}\right\rfloor \\
& x_{i} \in\{0,1\},
\end{array}
$$

and

$$
\begin{array}{ll}
\min & \sum_{i \in \mathrm{N}} \mu_{i} x_{i} \\
\text { s.t. } & \sum \mu_{i} x_{i} \geq\left\lceil\alpha z^{*}\right\rceil \\
& x_{i} \in\{0,1\} .
\end{array}
$$

A solution to (12) is given by $x^{*} \in\left\{x_{1}^{*}, x_{2}^{*}\right\}$ such that $Z_{\mathcal{R}}\left(x^{*}\right)=\max \left(Z_{\mathcal{R}}\left(x_{1}^{*}\right), Z_{\mathcal{R}}\left(x_{2}^{*}\right)\right)$.

Problems (17) and (18) are weakly polynomial, because they are particular cases of the knapsack problem. Finally, $\left\lfloor\alpha z^{*}\right\rfloor$ and $\left\lceil\alpha z^{*}\right\rceil$ can be computed in $O\left(\log _{2} \sum \mu_{i}\right)$, using a dichotomic search based on the sign of $\hat{Z}^{\prime}$.

\subsubsection{Fixed weights}

We show next a result similar to Theorem 2 when $a_{i}, i \in \mathrm{N}$, are fixed and $a_{0}$ is an arbitrary random variable.

Theorem 3 Consider the problem

$$
\max _{x \in \mathrm{B}} \sum a_{i} x_{i}-K \mathbb{E}_{a}\left[\max \left(0, \sum a_{i} x_{i}-a_{0}(\omega)\right)\right]
$$

where $a_{0}$ is a random variable. Problem (19) is weakly $\mathcal{N} \mathcal{P}$-hard and can be solved by a pseudo-polynomial algorithm in $O\left(n \sum a_{i}\right)$.

Proof The reduction from Proposition 1 holds when the variance of $a_{0}$ is zero and $K=\max _{i \in \mathrm{N}} a_{i}+1$. Let $Z$ be the objective function from (19). We must prove that $Z$ has the same properties as $Z_{\mathcal{R}}$ from previous section so that the same argument as in the proof of Theorem 2 can be applied:

- $Z$ depends only on $z=\sum a_{i} x_{i}: Z(x)=\hat{Z}(z)$.

$-\hat{Z}:\left[0, \sum a_{i}\right] \rightarrow \mathbb{R}$ is concave.

- We can compute $\left\lceil z^{*}\right\rceil$ and $\left\lfloor z^{*}\right\rfloor$ in $O\left(n \sum a_{i}\right)$.

It is straightforward to see that $Z$ depends only on $z=\sum a_{i} x_{i}$, with $\hat{Z}(z)=z-$ $\mathbb{E}_{a_{0}}\left[\max \left(0, z-a_{0}(\omega)\right)\right]$.

Then, the concavity of $Z$ (and therefore $\hat{Z}$ ) follows from a general result in stochastic programming [5]. Using $\delta>0$ small enough, we can use compute $\left\lceil z^{*}\right\rceil$ and $\left\lfloor z^{*}\right\rfloor$ by a dichotomic search based on the sign of $\hat{Z}(x)-\hat{Z}(x+\delta)$. 


\section{Algorithms and computational experiments}

In this section, we assume that random vector $a$ is absolutely continuous, with density function $f_{a}$. One of the main difficulty of (SCAP) is to evaluate the concave expectation term from (4):

$$
\begin{aligned}
\mathcal{Q}(x) & =-K \mathbb{E}_{a}\left[\max \left(0, \sum_{i=1}^{n} a_{i}(\omega) x_{i}-a_{0}(\omega)\right)\right] \\
& =-K \int_{A} f_{a}(a) \max \left(0, \sum_{i=1}^{n} a_{i} x_{i}-a_{0}\right) d a_{0} d a_{1} \ldots d a_{n} .
\end{aligned}
$$

For an arbitrary continuous random vector $a$, the multivariate integral (20) is nontrivial and must be solved using efficient packages for numerical integration, see [27]. To avoid this computational burden, we restrict ourselves to particular cases involving Gaussian and uniform distributions in the next subsections. Note that for special distributions, such as the exponential, the recourse function has a closed form [12].

\subsection{General case}

\subsubsection{LP/NLP algorithm}

Problem (SCAP) belongs to the class of mixed-integer non-linear programs, which have witnessed a tremendous attention in recent years. Efficient algorithms and solvers are now available to handle convex MINLP, see $[1,6,7]$. Moreover, results from [7] suggest that LP/NLP algorithms are particularly efficient to handle problems with linear constraints only, such as (SCAP). Notice that these particular LP/NLP algorithms turn out to be branch-and-cut algorithms where cuts are linearizations of the objective function. The main steps of our LP/NLP algorithm are explained in detail in what follows.

Consider the following linearly constrained convex MINLP:

$$
\begin{aligned}
\max & h(x) \\
\text { s.t. } & A x \leq b \\
& x_{i} \in\{0,1\},
\end{aligned}
$$

where $h$ is assumed concave and differentiable. Problem $(\mathrm{P})$ is equivalent to

$$
\begin{array}{ll}
\max & \gamma \\
\text { s.t. } & h(\bar{x})+\sum_{i=1}^{n} \frac{\partial h}{\partial x_{i}}(\bar{x})\left(x_{i}-\bar{x}_{i}\right) \geq \gamma \quad \bar{x} \in \mathbb{R}_{+}^{n} \\
& A x \leq b \\
& x_{i} \in\{0,1\},
\end{array}
$$

which has an infinite number of constraints. The main idea of outer-approximation is that, given a sensitivity parameter $\epsilon>0$, only a finite number of constraints (21) are 
required in a solution. Given a cut pool R, we define the upper bounding problem

$$
\begin{array}{ll}
\max & \gamma \\
\text { s.t. } & h(\bar{x})+\sum_{i=1}^{n} \frac{\partial h}{\partial x_{i}}(\bar{x})\left(x_{i}-\bar{x}_{i}\right) \geq \gamma \quad \bar{x} \in \mathrm{R} \\
& A x \leq b \\
& x_{i} \in\{0,1\} .
\end{array}
$$

Our algorithm lp/nlp solves (MP) with the branch-and-cut described in Algorithm 1. $\mathrm{T}$ represents the branch-and-cut tree and solving a node $o^{\prime} \in \mathrm{T}$ means solving the LP relaxation of (MP) augmented with branching constraints of $o^{\prime}$.

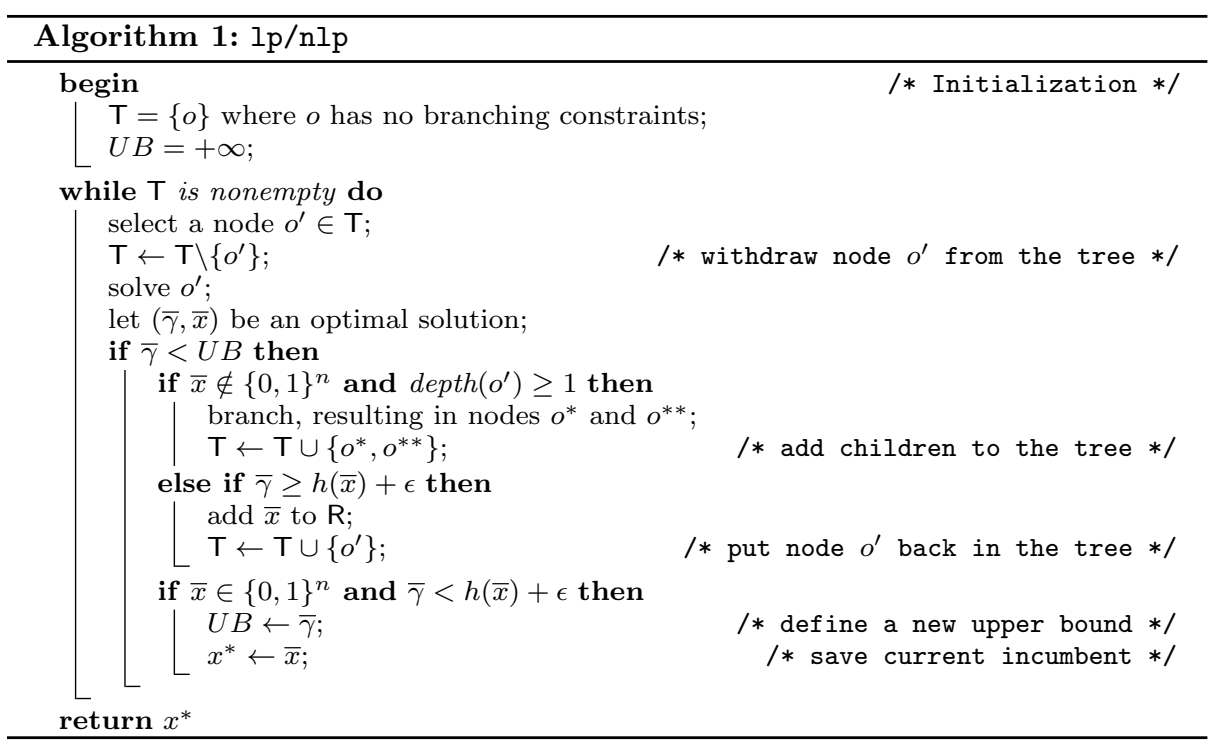

\subsubsection{Computational results}

We present next results of $1 \mathrm{p} / \mathrm{nl} \mathrm{p}$ for solving (SKP) with Gaussian weights and a fixed capacity. As previously mentioned, more general distributions could be handled as long as numerical integration packages are available. Recall that when each $a_{i}$ is a Gaussian $\mathcal{N}\left(\mu_{i}, \sigma_{i}\right)$ and $a_{0}$ is a constant, (SKP) can be rewritten as

$$
\max _{x \in B} \sum_{i \in N} p_{i} x_{i}-K\left\{\sigma(x) f\left(\frac{a_{0}-\mu(x)}{\sigma(x)}\right)+\left(\mu(x)-a_{0}\right) G\left(\frac{a_{0}-\mu(x)}{\sigma(x)}\right)\right\},
$$

where $\mu(x)=\sum_{i=1}^{n} \mu_{i} x_{i}, \sigma^{2}(x)=\sum_{i=1}^{n} \sigma_{i}^{2} x_{i}^{2}, f$ is the density function of $\mathcal{N}(0,1)$, $G=1-\Phi$ and $\Phi$ is the distribution function of $\mathcal{N}(0,1)$. Note that function $G$ is read from a table. Algorithm 1 is implemented within CPLEX 11 [13], with $\epsilon=0.1$. Since the model does not contain explicitly all constraints, we must deactivate the dual 
presolve, setting BooleanParam.PreInd to false. Then, we implemented our (global) cut generation with a LazyConstraintCallback, preventing CPLEX from using the dynamic search. The algorithm has been coded in JAVA on a HP Compaq 6510b with a processor Intel Core 2 Duo of $2.40 \mathrm{GHz}$ and $2 \mathrm{~GB}$ of RAM memory. We fix a time limit of 100 seconds per instance and the solution time has been set to 100 seconds for instances who could not be solved within this time limit or who exceeded the available memory.

We generated randomly different sets of instances, inspired by the instances from Martello et al. [22]. We consider two data ranges: $R=1000$ and $R=10000$. Then, parameters $\mu_{i}$ and $p_{i}$ for each item $i \in \mathrm{N}$ are integers uniformly generated between 4 and $R$. Each variance $\sigma_{i}$ is an integer uniformly generated between 1 and $\lfloor\mu / 4\rfloor$ for each item $i \in \mathrm{N}$, so that negative outcomes are negligible as explained next. We generated 100 instances for each value of parameters $K, n$ and $R$, with a capacity $a_{0}=(h / 101) \sum \mu_{i}$ for instance number $h$; all results take the average over the groups of 100 instances.

Note that Gaussian variables can take negative values which do not make sense in real applications. Nevertheless, when the ratio $\sigma / \mu$ is small enough, the probability that this happens is negligible so that it does not affect sensibly the objective. For instance, the probability that $\mathcal{N}\left(\mu, \sigma^{2}\right)$ takes a value less than $\mu-4 \sigma$ is slightly less than 0.0001 . Therefore we will assume $\mu / 4 \sigma \geq 1$ when generating our instances.

The following results show that the the penalty factor has little impact on the solution times, while a larger number of items makes the problem more difficult to solve. We study more specifically the time spent at the root node of Algorithm 1. The tables below report the total time in seconds (Time), the fraction of time spent at the root node (Initialization), the number of cuts generated at the root node and the number of cuts added deeper in the tree. We can see in Table 1 that the penalty factor $K$ has little

\begin{tabular}{c|cccccccc}
\hline \multirow{2}{*}{$K$} & \multicolumn{2}{|c}{ Time } & \multicolumn{2}{c}{ Initialization } & \multicolumn{2}{c}{ InitCuts } & \multicolumn{2}{c}{ AddCuts } \\
& $R=10^{3}$ & $R=10^{4}$ & $R=10^{3}$ & $R=10^{4}$ & $R=10^{3}$ & $R=10^{4}$ & $R=10^{3}$ & $R=10^{4}$ \\
\hline 2 & 0.346 & 0.332 & $65 \%$ & $65 \%$ & 8.33 & 8.26 & 0.69 & 0.73 \\
4 & 0.369 & 0.357 & $64 \%$ & $64 \%$ & 8.83 & 8.73 & 0.97 & 0.85 \\
6 & 0.358 & 0.357 & $64 \%$ & $65 \%$ & 8.98 & 8.98 & 0.98 & 0.78 \\
8 & 0.362 & 0.36 & $65 \%$ & $66 \%$ & 9.09 & 9.06 & 0.79 & 0.78 \\
10 & 0.343 & 0.341 & $64 \%$ & $63 \%$ & 9.15 & 9.18 & 0.8 & 0.91 \\
12 & 0.341 & 0.341 & $63 \%$ & $62 \%$ & 9.23 & 9.19 & 0.98 & 1.0 \\
14 & 0.341 & 0.379 & $65 \%$ & $65 \%$ & 9.33 & 9.28 & 0.79 & 0.84 \\
16 & 0.352 & 0.363 & $67 \%$ & $66 \%$ & 9.37 & 9.1 & 0.7 & 0.78 \\
18 & 0.381 & 0.361 & $66 \%$ & $64 \%$ & 9.46 & 9.14 & 0.76 & 0.86 \\
20 & 0.386 & 0.369 & $65 \%$ & $66 \%$ & 9.71 & 9.3 & 0.88 & 0.81 \\
\hline
\end{tabular}

Table 1 Uncorrelated Instances, $n=500$.

influence on lp/nlp. Therefore, we fix $K=10$ in the other tests. Results from Table 2 show that we can easily solve problems up to 5000 variables, even though times are significantly larger than in the deterministic case. For example, uncorrelated instances with 5000 items are solved by [22] on average in 0.01 seconds, whereas we need on average 19 seconds to solve such problems. Note that an important fraction of the time is spent in the generation of the cut pool at the root node, because most instances need to explore less than 100 nodes in their branch-and-cut trees. Pursuing our comparison with the deterministic knapsack, we wondered whether strongly correlated and Avis 


\begin{tabular}{|c|c|c|c|c|c|c|c|c|}
\hline \multirow[b]{2}{*}{$n$} & \multicolumn{2}{|c|}{ Time } & \multicolumn{2}{|c|}{ Initialization } & \multicolumn{2}{|c|}{ InitCuts } & \multicolumn{2}{|c|}{ AddCuts } \\
\hline & $R=10^{3}$ & $R=10^{4}$ & $R=10$ & $R=10^{4}$ & $R=10^{3}$ & $R=10^{4}$ & $R=10^{3}$ & $R=10^{4}$ \\
\hline 200 & 0.072 & 0.073 & $54 \%$ & $49 \%$ & 9.04 & 8.77 & 1.09 & 1.28 \\
\hline 400 & 0.209 & 0.193 & $65 \%$ & $62 \%$ & 9.08 & 8.75 & 0.98 & 0.95 \\
\hline 600 & 0.381 & 0.353 & $70 \%$ & $72 \%$ & 8.85 & 9.03 & 0.94 & 0.59 \\
\hline 800 & 0.616 & 0.579 & $75 \%$ & $77 \%$ & 8.96 & 8.97 & 0.66 & 0.49 \\
\hline 1000 & 0.896 & 0.876 & $78 \%$ & $78 \%$ & 8.73 & 8.92 & 0.61 & 0.61 \\
\hline 2000 & 3.447 & 3.361 & $83 \%$ & $84 \%$ & 8.81 & 9.2 & 0.5 & 0.42 \\
\hline 3000 & 7.329 & 7.5 & $86 \%$ & $87 \%$ & 8.85 & 9.22 & 0.34 & 0.28 \\
\hline 4000 & 13.302 & 12.682 & $86 \%$ & $87 \%$ & 8.93 & 8.9 & 0.42 & 0.25 \\
\hline 5000 & 20.405 & 17.462 & $86 \%$ & $86 \%$ & 9.02 & 7.8 & 0.4 & 0.28 \\
\hline
\end{tabular}

Table 2 Uncorrelated Instances, $K=10$.

instances [22] are harder to solve than uncorrelated ones. Strongly correlated instances are characterized by the relations $p_{i}=\mu_{i}+R / 10, i \in \mathrm{N}$, while Avis instances are defined as follows: $p_{i}$ is an integer uniformly generated between 1 and 1000, $\mu_{i}=$ $n(n+1)+i$, and $a_{0}=n(n+1)\lfloor(n-1) / 2\rfloor+n(n-1) / 2$. Results from Table 3 show that strongly correlated instances are roughly of the same difficulty as the uncorrelated ones, whereas strongly correlated ones are harder in the deterministic case. Avis instances are significantly harder to solve, see Table 4. Column (Unsolved) reports the number of unsolved instance within 100 seconds. While other problems were essentially solved a the root node, solving even small Avis required to spend a large amount of time exploring branch-and-cut trees. In fact, unreported results show that thousands of nodes where required to solve Avis instances, while uncorrelated and strongly correlated instances were usually solved by exploring less than a hundred of nodes.

\begin{tabular}{c|cccccccc}
\hline \multirow{2}{*}{$n$} & \multicolumn{2}{|c}{ Time } & \multicolumn{2}{c}{ Initialization } & \multicolumn{2}{c}{ InitCuts } & \multicolumn{2}{c}{ AddCuts } \\
& $R=10^{3}$ & $R=10^{4}$ & $R=10^{3}$ & $R=10^{4}$ & $R=10^{3}$ & $R=10^{4}$ & $R=10^{3}$ & $R=10^{4}$ \\
\hline 200 & 0.122 & 0.149 & $29 \%$ & $26 \%$ & 8.46 & 8.66 & 2.27 & 2.22 \\
400 & 0.353 & 0.374 & $33 \%$ & $31 \%$ & 8.64 & 8.6 & 1.86 & 1.94 \\
600 & 0.927 & 0.718 & $25 \%$ & $33 \%$ & 8.24 & 8.42 & 1.81 & 1.51 \\
800 & 0.842 & 1.064 & $47 \%$ & $36 \%$ & 8.23 & 8.16 & 1.17 & 1.33 \\
1000 & 2.09 & 1.006 & $30 \%$ & $57 \%$ & 8.05 & 8.16 & 0.89 & 0.99 \\
2000 & 3.155 & 2.727 & $76 \%$ & $78 \%$ & 7.91 & 7.85 & 0.59 & 0.4 \\
\hline
\end{tabular}

Table 3 Strongly Correlated Instances, $K=10$.

\begin{tabular}{c|ccccc}
\hline$n$ & Time & Unsolved & Initialization & InitCuts & AddCuts \\
\hline 200 & 6.639 & 0 & $0.23 \%$ & 11.34 & 1.79 \\
400 & 29.379 & 6 & $0.05 \%$ & 12.55 & 1.52 \\
\hline
\end{tabular}

Table 4 Avis Instances, $K=10$. 
4.2 Pseudo-polynomial cases

In Section 3 we proved that special cases of (SKP) can be solved in pseudo-polynomial time. Since we provide constructive proofs, a natural question is to find out whether the pseudo-polynomial algorithms proposed in the proofs perform better than $1 \mathrm{p} / \mathrm{nlp}$. Although we present results for (SKP) only, the algorithms described next could be used within decomposition schemes for more general problems of type (SCAP). For instance, a classical method to solve the generalized assignment problem consists in relaxing the demand constraints in a Lagrangian fashion, obtaining $m$ independent knapsack problems $[9,25]$. Similarly, one can relax constraints (5) in (SEGAP), yielding $m$ independent problems (SKP) that can be solved through dynamic programming whenever weights and capacity are distributed according to one of the assumptions from Section 3.

In all the following results, we fix a time limit of 100 seconds per instance and the solution time has been set to 100 seconds for instances which could not be solved within this time limit or which exceeded the available memory.

\subsubsection{Fixed weights and uniformly distributed capacity}

Lemma 2 shows how to obtain the solution to problem (8) from the solutions to two knapsack problems and to the problem of maximizing the concave and quadratic function of binary variables (11). The computational results from Martello et al. [22] show that the two knapsack problems can be solved in a very short amount of time, and we provide below results of $\mathrm{lp} / \mathrm{nl} \mathrm{p}$ applied to (11). Notice that other methods than $\mathrm{lp} / \mathrm{nl} \mathrm{p}$ can be used to solve (11). As mentioned in the proof of Lemma 5, (11) can be identified to a half-product that can be solved in pseudo-polynomial time. However, the algorithm from Badics and Boros performed much worse than $1 \mathrm{p} / \mathrm{nl}$, because very large numbers of states needed to be enumerated. Since the algorithm could hardly solve small instances with 200 variables, we do not report these computational experiments. Commercial MIP solvers are also able to handle (11). We provide below a numerical comparison of CPLEX 11 and $\mathrm{lp} / \mathrm{nl} \mathrm{p}$ described in Section 4.1. The instances are generated

\begin{tabular}{c|cc|cccc|cc}
\hline \multirow{2}{*}{$K$} & \multicolumn{2}{|c|}{$\begin{array}{c}\text { lp/nlp } \\
\text { Time }\end{array}$} & \multicolumn{2}{c|}{ Time } & \multicolumn{2}{c}{ cplex } & \multicolumn{2}{c}{ Time ratios } \\
& $R=10^{3}$ & $R=10^{4}$ & $R=10^{3}$ & $R=10^{4}$ & $R=10^{3}$ & $R=10^{4}$ & $R=10^{3}$ & $R=10^{4}$ \\
\hline 2 & 0.019 & 0.02 & 0.019 & 0.038 & 0 & 1 & 0.7 & 0.91 \\
4 & 0.018 & 0.02 & 0.036 & 0.943 & 0 & 0 & 0.78 & 0.82 \\
6 & 0.02 & 0.021 & 0.246 & 5.718 & 0 & 0 & 0.77 & 1.27 \\
8 & 0.019 & 0.023 & 1.311 & 9.913 & 0 & 1 & 1.05 & 0.59 \\
10 & 0.02 & 0.022 & 2.598 & 11.894 & 0 & 1 & 1.15 & 0.91 \\
12 & 0.02 & 0.022 & 3.233 & 14.764 & 0 & 1 & 1.11 & 0.94 \\
14 & 0.021 & 0.022 & 3.299 & 17.98 & 0 & 0 & 1.09 & 3.93 \\
16 & 0.021 & 0.023 & 3.329 & 21.403 & 1 & 0 & 1.29 & 6.15 \\
18 & 0.022 & 0.025 & 4.662 & 25.744 & 0 & 2 & 1.14 & 1.19 \\
20 & 0.021 & 0.024 & 5.256 & 28.096 & 0 & 2 & 1.23 & 1.39 \\
\hline
\end{tabular}

Table 5 Comparison between $1 \mathrm{p} / \mathrm{nlp}$ and $\mathrm{cplex}$ when $K$ increases, $n=100$.

as follows. The parameters $a_{i}$ and $p_{i}$ for each item $i \in\{1, \ldots, n\}$ are integers uniformly 
generated between 1 and $R$. For each data range $R$, each value of the penalty factor $K$ and number of items $n$, we generate 100 instances, with $\mathbb{E}_{a}\left(a_{0}\right)=(h / 101) \sum a_{i}$ for instance number $h$. Capacity $a_{0}$ varies uniformly between $90 \%$ of $\mathbb{E}_{a}\left(a_{0}\right)$ and $110 \%$ of $\mathbb{E}_{a}\left(a_{0}\right)$. We report on Tables 5 and 6 the average solution time in seconds and the number of unsolved instances for cplex only because $1 \mathrm{p} / \mathrm{nlp}$ could solve all of them within the time limit. Ratios, given by (Time cplex)/ (Time lp/nlp), are computed for each instance separately; we report the geometric average of the ratios, whereas we report the arithmetic average of solution times. We compute geometric average for ratios because of the following observation : if the ratio for one instance is $1 / 2$ and the one for another instance is 2 , their "average" should be equal to one, which is the case using the geometric average.

From Table 5, we see that the $\mathrm{lp} / \mathrm{nlp}$ performance does not depend on the value of $K$, and that instances for the two range values are of the same difficulty. However cplex requires more time to solve instances with $R=10^{4}$ than those with $R=10^{3}$, which becomes even more significant when $K$ increases. Even tough cplex takes on average more time than $l p / n l p$, the ratios close to one tell us that some instances are still solved faster by cplex than by $1 \mathrm{p} / \mathrm{nl}$. Table 6 studies the impact of increasing the number $n$

\begin{tabular}{|c|c|c|c|c|c|c|c|c|}
\hline \multirow[b]{2}{*}{$n$} & \multirow{2}{*}{\multicolumn{2}{|c|}{$\begin{array}{c}\text { lp/nlp } \\
\text { Time } \\
R=10^{3} R=10^{4}\end{array}$}} & \multicolumn{4}{|c|}{ cplex } & \multicolumn{2}{|c|}{ Time ratios } \\
\hline & & & $R=10^{\mathrm{T}}$ & $R=10^{4}$ & $\begin{array}{r}\mathrm{U} \\
R=1\end{array}$ & $\begin{array}{l}\mathrm{d} \\
=10^{4}\end{array}$ & & $R=10^{4}$ \\
\hline 200 & 0.029 & 0.032 & 0.08 & 0.269 & 0 & 0 & 1.93 & 1.77 \\
\hline 400 & 0.052 & 0.057 & 0.484 & 0.239 & 0 & 0 & 5.33 & 4.14 \\
\hline 600 & 0.076 & 0.081 & 1.878 & 0.638 & 1 & 0 & 9.51 & 7.75 \\
\hline 800 & 0.097 & 0.109 & 2.083 & 1.447 & 0 & 0 & 14.83 & 13.31 \\
\hline 1000 & 0.12 & 0.129 & 3.814 & 2.607 & 0 & 0 & 22.19 & 20.26 \\
\hline 1200 & 0.144 & 0.166 & 4.176 & 4.064 & 2 & 1 & 28.52 & 28.29 \\
\hline 1400 & 0.174 & 0.19 & 5.797 & 5.727 & 1 & 0 & 31.88 & 30.33 \\
\hline 1600 & 0.203 & 0.222 & 6.982 & 7.984 & 1 & 0 & 34.8 & 36.09 \\
\hline 1800 & 0.225 & 0.249 & 10.708 & 11.142 & 0 & 0 & 43.05 & 44.94 \\
\hline 2000 & 0.264 & 0.281 & 12.313 & 14.309 & 2 & 0 & 48.28 & 50.86 \\
\hline
\end{tabular}

Table 6 Comparison between $\mathrm{lp} / \mathrm{nlp}$ and cplex when $n$ increases, $K=2$.

of items, hence variables in the model. It is clear from the values of the ratios that the $l p / n l p$ handles better bigger instances than $c p l e x$ does, the ratio average increases more or less linearly with the number of items. This may be explained by the following observation: cplex deals with $O\left(n^{2}\right)$ variables so that its solution time is very impacted by $n$. On the other hand, the number of variables in $1 \mathrm{p} / \mathrm{nlp}$ only increases linearly with $n$, because this algorithm deals implicitly with the non linear objective. Then, because $K$ is small enough, solution times required by cplex to solve instances with $R=10^{3}$ are similar to those required to solve instances with $R=10^{4}$. We stopped our test to 2000 variables because cplex required almost all the available memory to solve these instances.

\subsubsection{Subset sum with gaussian weights}

Our last group of tests studies subset sum instances with Gaussian weights as defined in Theorem 2. Therefore, $\mu_{i}$ are (integer) uniformly distributed between 1 and $R$ and 
$\sigma_{i}=\sqrt{\lambda \mu_{i}}$ and $a_{0}$ is a constant random variable strictly positive. We have two algorithms at our disposal to solve these problems. As for general knapsack problem with Gaussian weights, we can use $1 \mathrm{p} / \mathrm{nlp}$ described in Section 4.1.1. Alternatively, using Theorem 2, we can solve these problems by the algorithm described in Algorithm 2. The later essentially solves two subset-sum problems using the method decomp from Pisinger [26], available at www.diku.dk/hjemmesider/ansatte/pisinger/. Algorithm 2 has been coded in $\mathrm{C}$ on the same computer as the one used for $1 \mathrm{p} / \mathrm{nlp}$. Table 7

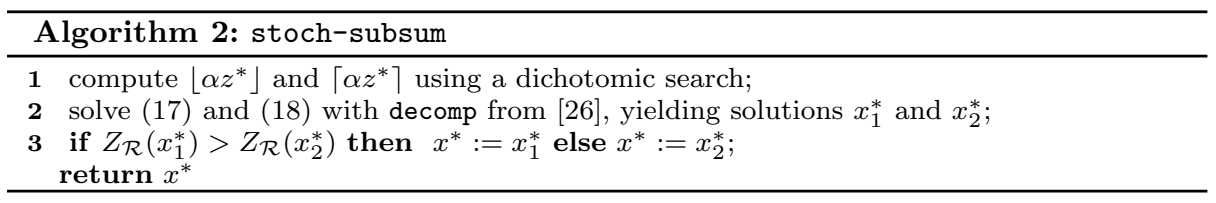

studies the sensibility to parameter $\lambda$ for $1 \mathrm{p} / \mathrm{nl} \mathrm{p}$, results for stoch-subsum are not reported because all problems are solved within 0.001 seconds. As expected, Theorem 2 enables us to solve subset sum problems orders of magnitude faster than using $l p / n l p$, which is even more striking in Table 8 below.

\begin{tabular}{c|cccccccc}
\hline \multirow{2}{*}{$\lambda$} & \multicolumn{2}{|c}{ Time } & \multicolumn{2}{c}{ Initialization } & \multicolumn{2}{c}{ InitCuts } & \multicolumn{2}{c}{ AddCuts } \\
& $R=10^{3}$ & $R=10^{4}$ & \multicolumn{2}{c}{$R=10^{3}$} & $R=10^{4}$ & $R=10^{3}$ & $R=10^{4}$ & \multicolumn{2}{c}{$R=10^{3}$} & $R=10^{4}$ \\
\hline $1 / 64$ & 1.783 & 1.979 & $7 \%$ & $5 \%$ & 11.14 & 7.03 & 2.11 & 1.42 \\
$1 / 16$ & 1.625 & 1.852 & $8 \%$ & $3 \%$ & 10.9 & 11.19 & 1.58 & 1.63 \\
$1 / 4$ & 1.609 & 1.891 & $6 \%$ & $3 \%$ & 10.42 & 10.97 & 1.45 & 1.96 \\
\hline
\end{tabular}

Table 7 Subset Sum with $n=500$ and $K=10$.

We fix $\lambda=1 / 16$ in our subsequent computational results. Table 8 compares $1 \mathrm{p} / \mathrm{nlp}$ and stoch-subsum for different values of $n$. Whenever stoch-subsum was able to solve an instance in less than 0.001 seconds, its solution time was set to 0.001 seconds. Ratios, given by (Time lp/nlp) / (Time stoch-subsum), are computed for each instance separately; we report the geometric average of the ratios, whereas we report the arithmetic average of solution times. Comparing Table 8 with Tables 2 and 3 , we see that stochastic subset sum problems are significantly harder to solve than uncorrelated and correlated instances. This is due to the size of the branch-and-cut trees, hundreds of nodes being explored for the subset sum instances.

Similarly to the Avis knapsack instances, we can define Avis subset sum instances as follows: $p_{i}=\mu_{i}=n(n+1)+i$, and $a_{0}=n(n+1)\lfloor(n-1) / 2\rfloor+n(n-1) / 2$. To obtain groups of 100 "different" instances, we shuffle the order in which the items are read. Avis subset sum are extremely hard to solve already in the deterministic case, specialized algorithms are needed to solve large instances. Table 9 compares $1 \mathrm{p} / \mathrm{nl} \mathrm{p}$ and stoch-subsum on these difficult instances. In fact, $1 \mathrm{p} / \mathrm{nlp}$ can not solve problem with more than 20 variables within the time limit of 100 seconds. This is due to the very large number of nodes explored. For $n=10$, lp/nlp explores around 751 nodes on average, while exactly 705430 nodes are explored for each instance with $n=20$. Although stoch-subsum requires more time than for other subset sum problems, it can still handle problems containing up to 600 variables within 100 seconds of CPU time. 


\begin{tabular}{|c|c|c|c|c|c|c|c|c|}
\hline \multirow[b]{2}{*}{$n$} & \multicolumn{4}{|c|}{ lp/nlp } & \multirow{2}{*}{\multicolumn{2}{|c|}{$\begin{array}{c}\text { stoch-subsum } \\
\text { Time } \\
R=10^{3} R=10^{4}\end{array}$}} & \multirow{2}{*}{\multicolumn{2}{|c|}{$\begin{array}{c}\text { Time ratios } \\
R=10^{3} R=10^{4}\end{array}$}} \\
\hline & $R=10^{3}$ & $R=10^{4}$ & $R=1$ & $\begin{array}{l}\text { lved } \\
R=10^{4}\end{array}$ & & & & \\
\hline 200 & 0.199 & 0.244 & 0 & 0 & 0.001 & 0.001 & 170 & 198 \\
\hline 400 & 0.917 & 0.991 & 0 & 0 & 0.001 & 0.001 & 636 & 736 \\
\hline 600 & 2.542 & 2.726 & 0 & 0 & 0.002 & 0.002 & 1331 & 1364 \\
\hline 800 & 5.306 & 5.568 & 0 & 0 & 0.002 & 0.002 & 2190 & 2096 \\
\hline 1000 & 10.12 & 9.716 & 0 & 0 & 0.003 & 0.003 & 3422 & 3085 \\
\hline 2000 & 84.768 & 79.023 & 31 & 26 & 0.005 & 0.005 & 12660 & 11475 \\
\hline
\end{tabular}

Table 8 Comparison of $1 \mathrm{p} / \mathrm{nlp}$ and stoch-subsum on subset sum instances with $K=10$ and $\lambda=1 / 16$.

\begin{tabular}{c|ccc}
\hline$n$ & lp/nlp & stoch-subsum & Time ratios \\
\hline 10 & 0.055 & 0.001 & 51.8 \\
20 & 40.124 & 0.001 & 40119 \\
100 & - & 0.055 & - \\
200 & - & 1.003 & - \\
300 & - & 5.22 & - \\
400 & - & 16.6 & - \\
500 & - & 41.6 & - \\
600 & - & 88.1 & - \\
\hline
\end{tabular}

Table 9 Comparison of $1 \mathrm{p} / \mathrm{nlp}$ and stoch-subsum on Avis subset sum instances with $K=10$ and $\lambda=1 / 16$.

\section{Conclusion}

In this paper, we study two important aspects of the stochastic knapsack problem with penalty recourse. We provide complexity results for three special cases of the problem, and propose a branch-and-cut algorithm for the general problem. The algorithm is tested on a large test of instances randomly generated and inspired by the instances used in [22] for the deterministic knapsack problem. We can solve in less than a minute uncorrelated and correlated problems involving up to 5000 variables. Only a few linearizations are required and less than 100 nodes are explored in the branch-and-cut tree. Similarly to the deterministic situation, Avis instances are very hard to solve, requiring the exploration of much larger trees.

The branch-and-cut algorithm is less efficient with subset sum problems, usually exploring up to a thousand of nodes. These subset sum problems can however be solved almost as fast as the deterministic version of the problem, by using a specialized algorithm from Pisinger [26]. The pseudo-polynomial algorithm devised for the knapsack problem with constant weights and capacity uniformly distributed is also tested, but does not provide positive results.

An interesting subject for future research would be to study how to extend efficiently the methods described herein to handle more general problems. We should evaluate numerically our branch-and-cut algorithm $\mathrm{lp} / \mathrm{nlp}$ for more general (SCAP), and test decomposition algorithms that reduce general problems to a sequence of knapsack problems. 


\section{Acknowledgements}

The authors thank the editor and an associate editor, whose valuable and constructive remarks improved the presentation of this paper.

\section{References}

1. K. Abhishek, S. Leyffer, and J. T. Linderoth. Filmint: An outer-approximation-based solver for nonlinear mixed integer programs. INFORMS Journal on computing. In press.

2. T. Badics and E. Boros. Minimization of half-products. Math. Oper. Res., 23(3):649-660, 1998

3. C. Barnhart, C. A. Hane, and P. H. Vance. Using branch-and-price-and-cut to solve origindestination integer multicommodity flow problems. Oper. Res., 48(2):318-326, 2000.

4. P. Beraldi and M. E. Bruni. An exact approach for solving integer problem under probabilistic constraints with random technology matrix. Annals of Operations Research, 177:127-137, 2010.

5. J. R. Birge and F. V. Louveaux. Introduction to Stochastic programming (2nd edition). Springer Verlag, New-York, 2011.

6. P. Bonami, L. T. Biegler, A. R. Conn, G. Cornuéjols, I. E. Grossmann, C. D. Laird, J. Lee, A. Lodi, F. Margot, N. Sawaya, and A. Wächter. An algorithmic framework for convex mixed integer nonlinear programs. Discrete Optimization, 5(2):186-204, 2008.

7. P. Bonami, M. Kilinc, and J. Linderoth. IMA Volumes. University of Minnesota, 2010. Algorithms and Software for Convex Mixed Integer Nonlinear Programs.

8. G.G. Brown and G.W. Graves. Real-time dispatch of petroleum tank trucks. Management Science, 27:19-32, 1981.

9. D. G. Cattrysse and L. N. Van Wassenhove. A survey of algorithms for the generalized assignment problem. European Journal of Operational Research, 60(3):260 - 272, 1992.

10. A. Cohn and C. Barnhart. The stochastic knapsack problem with random weights: A heuristic approach to robust transportation planning. In Proceedings of the Triennial Symposium on Transportation Analysis (TRISTAN III), 1998.

11. W.K. Klein Haneveld, L. Stougie, and M.H. van der Vlerk. Stochastic integer programming with simple recourse. Technical Report Research Memorandum 455, University of Groningen, 1991.

12. B. Hansotia. Some special cases of stochastic programs with recourse. Operations Research, 25(2):361-363, 1977.

13. ILOG CPLEX Division, Gentilly, France. ILOG. ILOG CPLEX 11.0 Reference Manual., 2007 .

14. A. J. Kleywegt and J. D. Papastavrou. The dynamic and stochastic knapsack problem with random sized items. Oper. Res., 49(1):26-41, 2001.

15. A. J. Kleywegt, A. Shapiro, and T. Homem de Mello. The sample average approximation method for stochastic discrete optimization. SIAM J. Optim., 12(2):479-502, 2002.

16. O. Klopfenstein. Solving chance-constrained combinatorial problems to optimality. Computational Optimization and Applications, In press.

17. O. Klopfenstein and D. Nace. A note on polyhedral aspects of a robust knapsack problem. Optimization Online, 2007.

18. O. Klopfenstein and D. Nace. A robust approach to the chance-constrained knapsack problem. Oper. Res. Lett., 36(5):628-632, 2008.

19. S. Kosuch and A. Lisser. Upper bounds for the 0-1 stochastic knapsack problem and a b\&b algorithm. Ann. Oper. Res., 2009. Article in press.

20. G. Laporte, F. V. Louveaux, and H. Mercure. The vehicle routing problem with stochastic travel times. Transp. Science, 26(3):161-170, 1992

21. F. V. Louveaux and M. H. van der Vlerk. Stochastic programming with simple integer recourse. Mathematical Programming, 61:301-325, 1993.

22. S. Martello, D. Pisinger, and P. Toth. Dynamic programming and strong bounds for the 0-1 knapsack problem. Management Science, 45(3):414-424, 1999.

23. D. P. Morton and R. K. Wood. Advances in computational and stochastic optimization, logic programming and Heuristic Search, chapter 5, pages 149-168. Woodruff, 1998.

24. J. M. Mulvey, R. J. Vanderbei, and A. Z. Stavros. Robust optimization of large-scale systems. Oper. Res., 43(2):264-281, 1995. 
25. R. M. Nauss. Solving the generalized assignment problem: An optimizing and heuristic approach. INFORMS J. on Computing, 15(3):249-266, 2003.

26. D. Pisinger. An exact algorithm for large multiple knapsack problems. European Journal of Operational Research, 114(3):528 - 541, 1999.

27. A. Prékopa. Stochastic Programming. Kluwer, 1995.

28. I. Quesada and I. E. Grossman. An LP/NLP based branch and bound algorithm for convex MINLP optimization problems. Comput. Chem. Eng., 16(10/11):937-947, 1992.

29. D. Spoerl and R.K Wood. A stochastic generalized assignment problem. INFORMS Annual Meeting, Atlanta, GA, 1922 October, 2003.

30. R.J.B. Wets. Solving stochastic programss with simple recourse. Stochastics, 10(3):219$242,1983$. 